\title{
"EPIDERMOID CYST OF HARD PALATE AND EVALUATION OF HEALING BY COLOR POWER DOPPLER ULTRASONOGRAPHY: A CASE REPORT
}

\author{
Sanjeev Chauhan ${ }^{1}$, Ashu Gupta ${ }^{2}$, Vishal Sharma ${ }^{3}$, Neeti Aggarwal ${ }^{4}$ \\ ${ }^{1}$ Post graduate Student, Deptt. of Conservative Dentistry and Endodontics, H.P.G.D.C., Himachal Pradesh, India \\ ${ }^{2}$ Professor and Head, Deptt. of Conservative Dentistry and Endodontics, H.P.G.D.C., Himachal Pradesh, India \\ ${ }^{3}$ Professor Deptt. of Conservative Dentistry and Endodontics H.P.G.D.C., Himachal Pradesh, India \\ ${ }^{4}$ Assistant Professor, Deptt. of Radiodiagnosis, I.G.M.C. Himachal Pradesh, India
}

\begin{abstract}
(
ABSTRACT

Epidermoid cyst is a rare developmental cyst of the oro-facial region which results from entrapped epidermal elements with an incidence of $6.9-7 \%$ and represents less than $0.01 \%$ of all oral cavity cysts. PRF, as a physiologic fibrin matrix, serves as a net to stem cells, especially when an accelerated angiogenesis develops in the fibrin membrane. This aspect is of particular interest in the case of wide osseous defects. The Color Power Doppler detects minute areas of blood flow and the velocity of blood flow in healing bone by recording the change in frequency caused by the moving red blood cells. It demonstrates the progressive formation of new vessels in bone during the initial healing period. As the bone remodelling proceeds, there is a decrease in flow signals.
\end{abstract}

Keywords: Epidermoid Cyst, PRF, MTA, Color Power Doppler Ultrasonography

\section{INTRODUCTION}

Epidermoid cysts are rare lesions of non-odontogenic nature inclusion cysts lined by ectoderm and derived from germinal epithelium and can be encountered throughout the body, in areas where embryonic elements fuse together. Most cases have been reported in ovaries and the testicles, with $7 \%$ occurring in the oro-facial area and $1.6 \%$ in the oral cavity, representing $0.01 \%$ of all oral cavity cysts. $^{2}$

In recent years autologous platelet concentrates have been used with the aim of enhancing the healing rate in bone regeneration procedures. Autologous Platelet rich fibrin (PRF) is a fibrin matrix

Corresponding Author: Sanjeev Chauhan E-mail: sanjeev1231971@gmail.com Received: $30^{\text {th }}$ December 2016

Accepted: $2^{\text {nd }}$ April 2017 Online: $20^{\text {th }}$ May 2017 in which platelet cytokines, growth factors, and cells are trapped which may be released after a certain time and can serve as a resorbable membrane. ${ }^{3}$ Choukroun and his associates were amongst the pioneers for using PRF protocol.

MTA is a calcium silicate -based hydraulic cement that possesses favourable biological properties including sealing ability, good biocompatibility and hard tissue -inducing effects. ${ }^{4}$

Ultrasound as a diagnostic tool has been widely used in many medical fields, and its applications in dentistry have not been sufficiently explored. US imaging provides sufficient information regarding the nature of the periapical lesions, and is a reliable diagnostic technique for differentiating periapical lesions. (Cotti et.al., 2006) $)^{5}$ 


\section{CASE REPORT}

A 35-year-old male patient reported to the Department of Conservative Dentistry and Endodontics with the chief complaint of draining pus from anterior palatal region. He had a history of swelling in the palate for past 10 years. Clinically, 21, 22 were discoloured and EPT showed them to be nonvital. A soft diffuse swelling was present in anterior palatal region crossing the midline slightly (Figure 1).

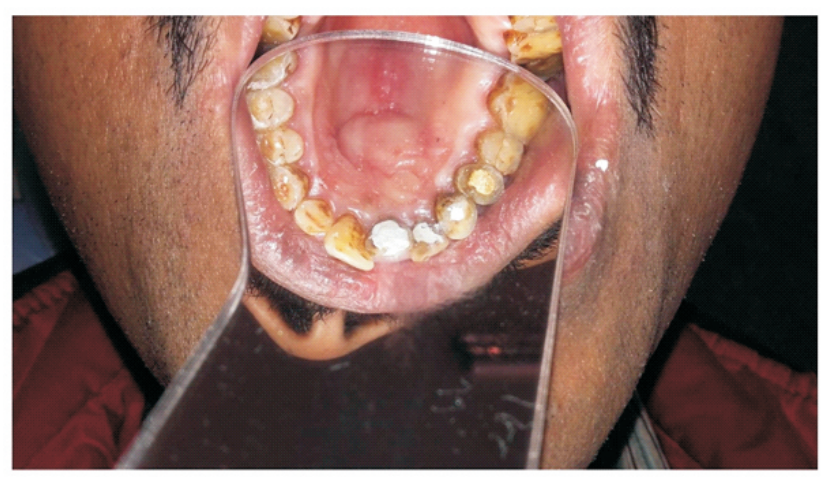

Figure 1: Swelling Present in Palate

The differential diagnosis included palatal abscess, traumatic or irritational fibroma, odontogenic or nonodontogenic cysts and tumours.

Upon radio graphic evaluation by IOPA and occlusal view, a large periapical radiolucency was seen at the apex of 21,22 . On opening the access cavity of 21 and 22 there was viscous discharge drainage from the canals. Subsequently debridement and irrigation was done with $3 \% \mathrm{NaOCl}$. Multiple visit endodontic treatment was performed with 21,22 and calcium hydroxide placed as an intra canal medicament for 3 weeks. The swelling decreased slightly but even after repeated dressing's purulent discharge continued. Then it was decided to do surgical excision of the lesion and fill the bony cavity with PRF. Ultrasonographic examination with Color Power Doppler was done preoperatively for evaluation of nature of lesion by an experienced radiologist using an ultrasonography machine with an endocavitary probe covered by an impervious plastic barrier sheath at 8-12 $\mathrm{M} \mathrm{Hz}$. and the following lesion parameters were checked by ultrasonography.

- Dimensions of the periapical lesion.

- Volume of the lesion.

- Type of echoes from the lesion and was found hypo echoic (pathological) in nature.

- Absence of vascularity in the lesion.

For the surgical procedure, under strict aseptic conditions full thickness flap was reflected by a sulcular incision with disposable B.P. blade no. 15 under local anaesthesia (xylocaine 2\% with adrenaline 1:80,000). A labial rectangular and palatal crevicular incision was given and full thickness mucoperiosteal flap was raised. Complete cystic enucleation was donefrom the palatal side (Figure 2). Apical resection was done with slow speed micromotor handpiece with

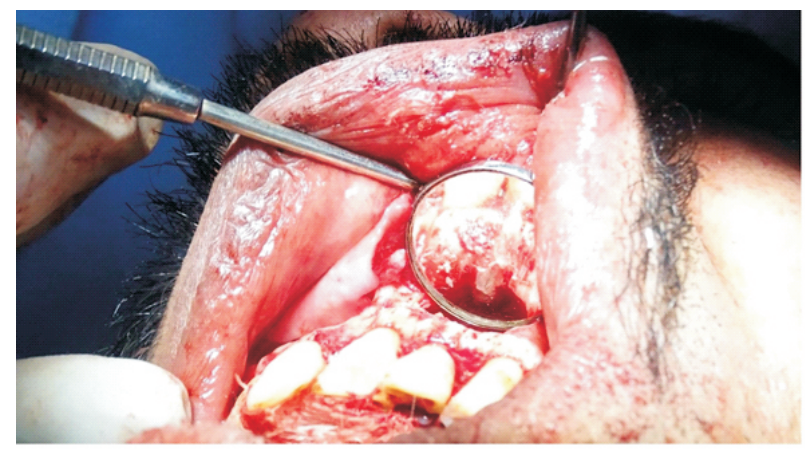

Figure 2: MTA Placed as Apical Seal

adequate normal saline irrigation and 2-3 $\mathrm{mm}$. of apex was removed from root end. Then proultra ultrasonic endodontic tip no. 1 was used to prepare the cavity in apical end of root end for MTA as apical seal. A minimum of 2-3 mm of cavity was prepared at root apex to provide the adequate seal followed by retrograde filling with white MTA (ANGELIUS) (Figure 3). Then a wet gauge piece was placed over MTA (10 minutes). The initial setting time of MTA ANGELIUS is 10 minutes and final setting time is 15 minutes.

PRF derived by Choukroun's technique $(20 \mathrm{ml}$. of patient's blood was collected without any anticoagulant 
and immediately centrifuged at $3000 \mathrm{rpm}$ for 10 minutes) was removed from the tube and the attached red blood cells scraped off and discarded. Then PRF

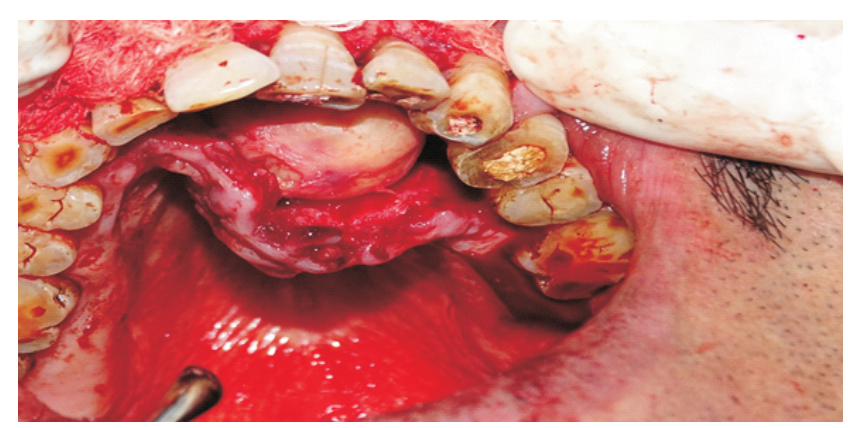

Figure 3: Cystic Lesion Before Excision

was pressed to form membrane and placed in the bony defect (Figure 4). The flap was reseated and sutured with non absorbable silk suture (3-0). Enucleated cystic lesion (Figure 5) was send for histopathological examination.

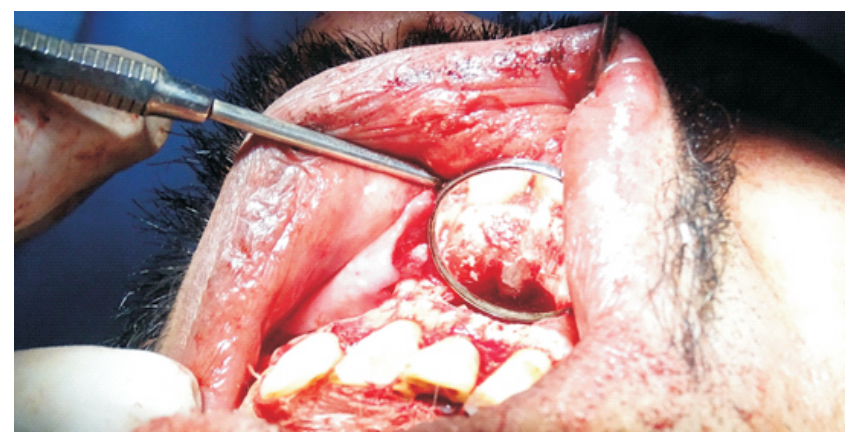

Figure 4: MTA Placed as Apical Seal

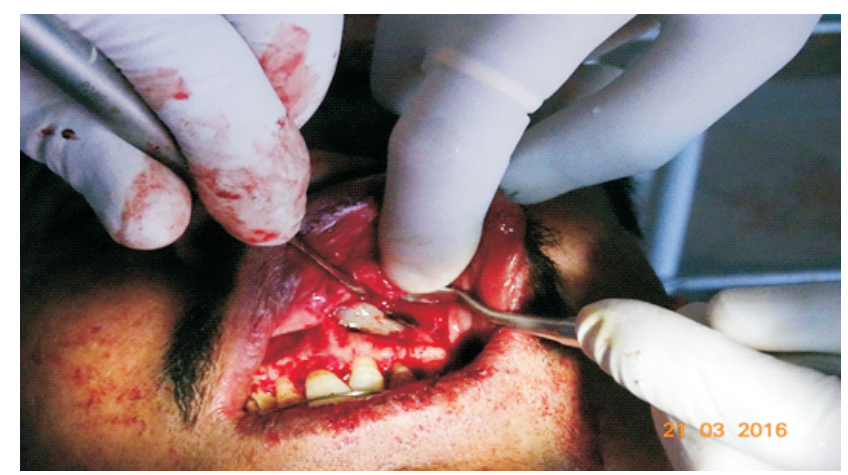

Figure 5: During Prf Placement

The labial and palatal flaps were sutured. Palatal splint was given to support the palatal soft tissues after surgery. Post-operatively antibiotics were prescribed for three days along with analgesics and postoperative instructions were given. Sutures were removed at $5^{\text {th }}$ postoperative day. The histopathological examination of the specimen (Figure 6)

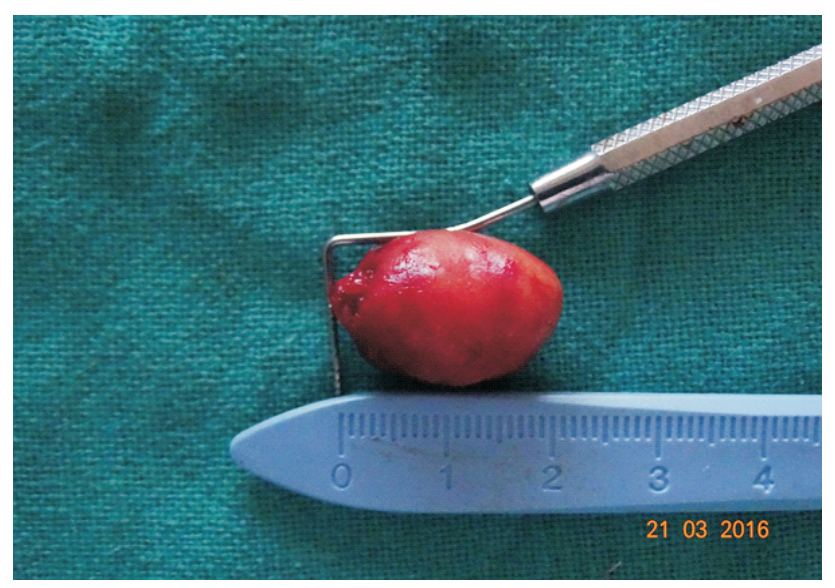

Figure 6: Excised Cystic Lesion

showed cyst lined by stratified squamous epithelium filled with keratin and epithelium showing granular layer confirming the diagnosis of nonodontogenic type of cyst as epidermal cyst.

The patient was followed-up after 1, 3 and 6 months postoperatively and evaluated clinically, radiographically and by Ultrasonographic examination. There was no sign or symptom of reccurence and lesion parameters were checked by Ultrasonographic examination with Color Power Doppler .It was found at each subsequent review that the lesion size consistently decreased in size showing presence of arterial vascularity at 6 months.

\section{DISCUSSION}

In the oral cavity Epidermoid cyst is mainly found in the floor of the mouth and is the most common location, and occasional occurrence have been reported involving buccal mucosa, tongue, lips, and uvula. Occurrence of Epidermoid cyst involving maxillary hard palate region is an unusual location. Epidermoid cysts are generally diagnosed in young adults in the second and third decades of life. It is twice 
as common in men as in women with a male to female ratio of $3: 1$.

Depending on the pathogenesis, Epidermoid cyst can be divided into:

1) Congenital

2) Acquired

Acquired cysts are derived from traumatic or iatrogenic inclusion of epithelial cells or from occlusion of sebaceous gland duct, it was first recognized by Werhner in 1855 and Sutton in 1895 referred it to be "Implantation cyst". 6

PRF contains all key immune cytokines such as interleukins IL1, IL-6,IL-4 and tumour necrosis factor. PRF has a supportive effect on the immune system and acts by stimulating defense mechanisms. This could be important in the case of wound infection. ${ }^{8}$ Acc. to Joseph Choukroun, et. al. PRF permits a rapid angiogenesis and an easier remodelling of fibrin in a more resistant connective tissue.

In PRF platelets and leukocyte cytokines play an important part and the fibrin matrix supporting them is responsible for the therapeutic potential of PRF. ${ }^{7}$ A progressive polymerization mode indicates increased incorporation of the circulating cytokines in the fibrin meshes. PRF, thus would be able to release cytokines during fibrin matrix remodelling and has a long term effect. $^{9}$

In the present study the healing potential of PRF was evaluated at varying intervals both qualitatively and quantitatively by means of color power doppler ultrasonography using MTA as apical seal.

Jessie F. Reyes-Carmona, et. al., studied ability of mineral trioxide aggregate (MTA) to promote hardtissue deposition and wound healing and concluded that MTA induced a pro inflammatory and pro wound healing environment. The bio mineralization process occurred simultaneously and promoted the integration of the biomaterial into the environment.

Namita Raghav,et.al., evaluated the efficacy of conventional radiography, digital radiography and ultrasound imaging in diagnosing periapical lesions.
The percentage accuracy of diagnosing periapical lesions using conventional radiography was $47.6 \%$, digital radiography $55.6 \%$, and ultrasound $95.2 \%$ according to them Ultrasound had the highest sensitivity and specificity.

Smita Singh,et.al.,(2013) in their case study also have observed and stated that it requires around 1 year for complete healing to occur after the periapical surgery while with the use of PRF, healing is fastened and requires approx 6 months for complete regeneration of bone similar to observations of our study. They also used PRF as matrix for faster bone formation and observed healing by ultrasonography.

The color power Doppler detects minute areas of blood flow and the velocity of blood flow in healing bone by recording the change in frequency caused by the moving red blood cells. In the present study vascularity (arterial) was absent in the lesion preoperatively but was evident at 6 months and peak systolic velocity was found to be $13.4 \mathrm{~cm} / \mathrm{s}$ (Figure 7 11).

In the study of healing in this case study it was observed that there was a consistent decrease in volume of lesion at all subsequent reviews postoperatively.

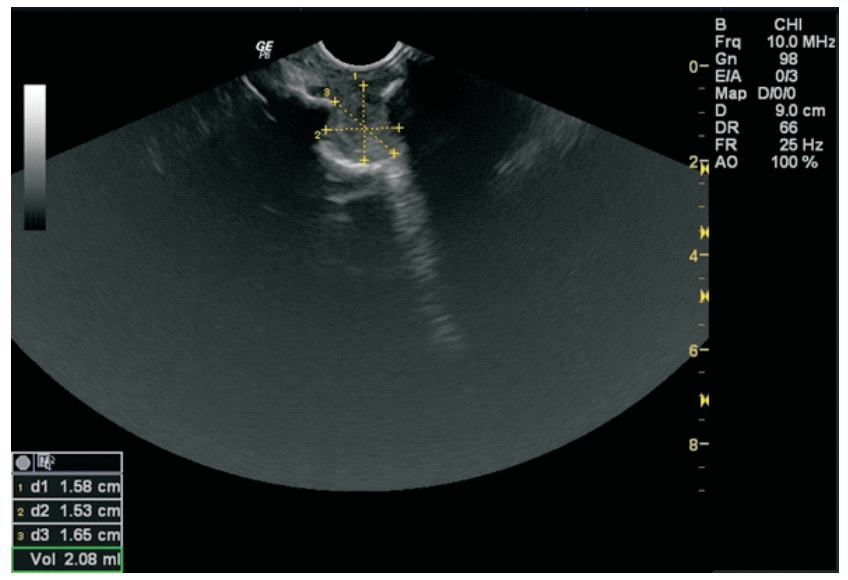

Figure 7: Ultrasonographic examination showing presurgical lesion size

Moreover as epidermoid cysts are kerain filled cystic lesions the ultrasonographic examination showed preoperative lesion to be hyperechoic in nature. Postoperatively at one month review the nature of 


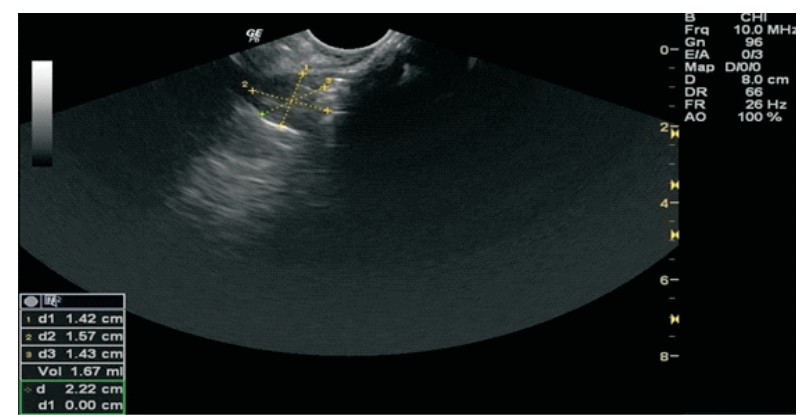

Figure 8: Lesion size at one month postoperatively

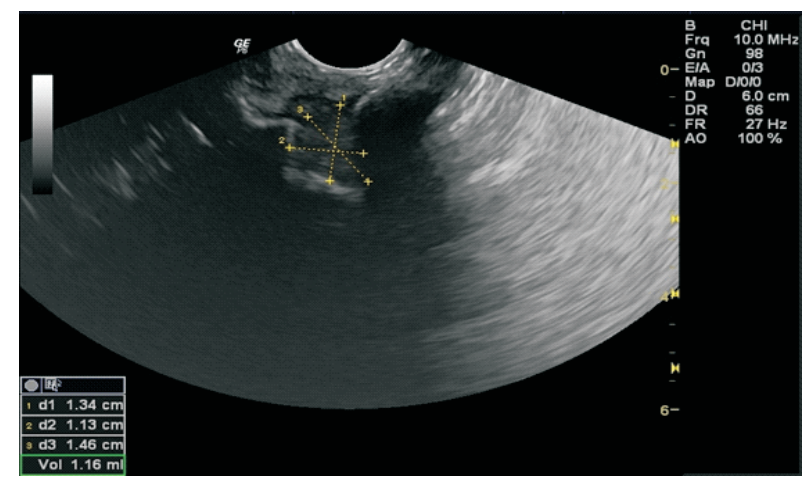

Figure 9: Lesion size at three months postoperatively

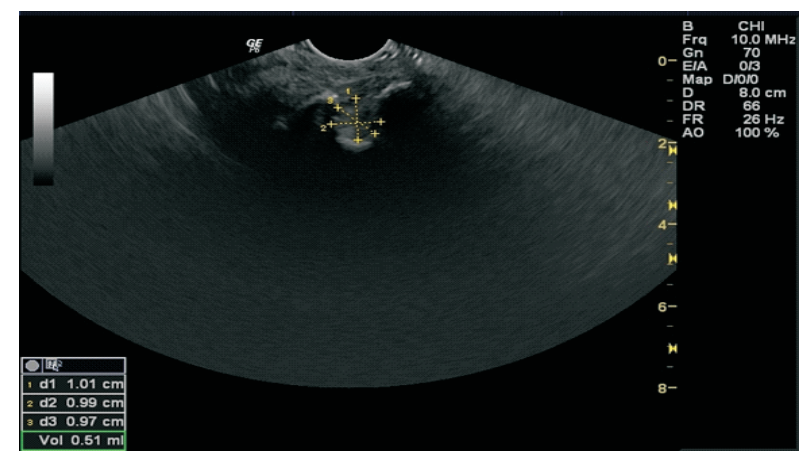

Figure 10: Lesion size at six months postoperatively

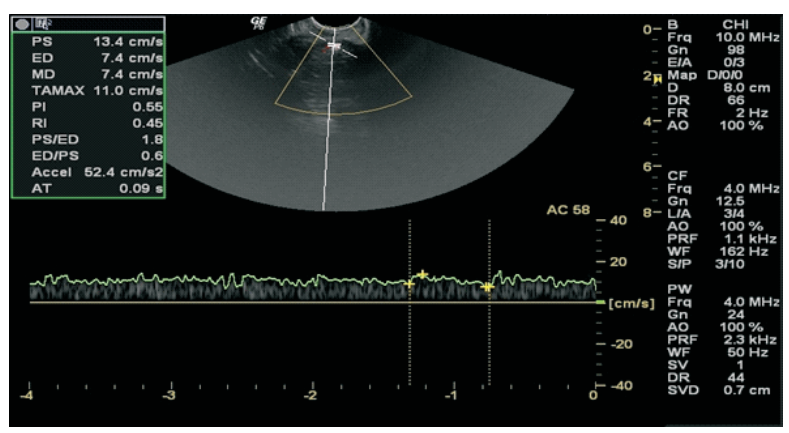

Figure 11: Presence of vascularity at six months

lesion was hypoechoic subsequently changing to predominantly hyperechoic nature at 6 months review as the healing progressed.

\section{CONCLUSION}

As per our study conclusion Epidermal cyst is a rare lesion of hard palate and PRF can be used for faster healing of such cases and Color Power Doppler Ultrasonography is an important and reliable tool to measure healing of such lesion using different parameters.

\section{REFERENCES}

1) Varun Rastogi, Naveen Puri, Geetpriya Kaur, Lalita Yadav, Rachna Sharma;Unusual Cases of Epidermoid cyst: Case Series, International Journal of Scientific Study , 2013; 01; (02); 72-75

2) Ozan F, Polat HB, Ay S, Goze F. Epidermoid cyst of the buccal mucosa: Acase report. J Contemp, Dent Pract 2007;8(3):1-6.

(3) Joseph Choukroun, David M. Dohan, Antoine Diss, Steve L. Dohan, Anthony J. J. Dohan, Jaafar Mouhyi, and Bruno Gogly, Platelet-rich fibrin (PRF): A second-generation platelet concentrate. Part II: Platelet-related biologic features Oral Med Oral Pathol Oral Radiol Endod 2006;101:E45-50

(4) Jessie F. Reyes-Carmona, Host-Mineral Trioxide Aggregate Inflammatory Molecular Signaling and Biomineralization Ability; JOE; 2010;36,(8);1347-1353

5) Namita Raghav, Sujatha S. Reddy, A. G. Giridhar, Srinivas Murthy, Yashodha Devi B. K, N. Santana, N. Rakesh, and Atul Kaushik, India Comparison of the efficacy of conventional radiography, digital radiography, and ultrasound in diagnosing periapical lesions Oral Surg.Oral Med. Oral Patho. Oral Radiol. Endod. 2010; 110:379-385

6) Noffke CE. Implantation-type epidermoid cyst of the mandible. Dento maxillofac Radiology 1999; 28: 383-385.

7) Choukroun J, Diss A, Simonpieri A, Girard MO, Schoeffler C, Dohan SL, Platelet-rich fibrin (PRF): A second-generation platelet concentrate. Part IV: Clinical effects on tissue healing. Oral Surg Oral Med Oral Pathol Oral Radiol Endod, 2006;101:e56-60

8) Dohan DM, Choukroun J, Diss A, Dohan SL, Dohan AJ, Mouhyi J. Platelet-rich fibrin (PRF): A second-generation platelet concentrate. Part II: Platelet-related biologic features. Oral Surg Oral Med Oral Pathol Oral Radiol Endod 2006;101:e45-50.

9) Choukroun J, Diss A, Simonpieri A, Girard MO, Schoeffler C, Dohan SL, Dohan AJ, Mouhyi J, Dohan DM. Platelet-rich fibrin (PRF): A second-generation platelet concentrate. Part V: histologic evaluations of PRF effects on bone allograft maturation in sinus lift. Oral Surg Oral Med Oral Pathol Oral Radiol Endod 2006;101:299-303.

Source of Support: Nil, Conflict of Interest: None Declared 